\title{
Numerical simulations of sessile droplet evaporating on heated substrate
}

\author{
Xue Chen ${ }^{1,2}$, Paul G. Chen ${ }^{2, a}$, Jalil Ouazzani ${ }^{3}$, and Qiusheng Liu ${ }^{1}$ \\ 1 Institute of Mechanics, Chinese Academy of Sciences, 100190, Beijing, P.R. China \\ 2 Aix Marseille Univ., CNRS, Centrale Marseille, M2P2, Marseille, France \\ 3 Arcofluid Consulting LLC, 309 N Orange Ave, Orlando, FL 32801, USA
}

Received 30 June 2016 / Received in final form 31 July 2016

Published online 2 May 2017

\begin{abstract}
Motivated by the space project EFILE, a 2D axisymmetric numerical model in the framework of ALE method is developed to investigate the coupled physical mechanism during the evaporation of a pinned drop that partially wets on a heated substrate. The model accounts for mass transport in surrounding air, Marangoni convection inside the drop and heat conduction in the substrate as well as moving interface. Numerical results predict simple scaling laws for the evaporation rate which scales linearly with drop radius but follows a power-law with substrate temperature. It is highlighted that thermal effect of the substrate has a great impact on the temperature profile at the drop surface, which leads to a multicellular thermocapillary flow pattern. In particular, the structure of the multicellular flow behavior induced within a heated drop is mainly controlled by a geometric parameter (aspect ratio). A relationship between the number of thermal cells and the aspect ratio is proposed.
\end{abstract}

\section{Introduction}

Evaporation of a sessile liquid drop is a common phenomenon in nature. It has many important practical applications in areas including industry, biomedicine and space thermal devices which involve MEMS cooling, DNA mapping, thin film coating and micro heat pipes [1]. Therefore, there has been a rapid growth of scientific and technological interest in the problem of droplet evaporation $[2,3]$.

The physical process which controls the complex problem of diffusion, Marangoni convection and heat conduction has been the subject of several recent reviews. $\mathrm{Hu}$ and Larson [4] used a finite-element method to solve the diffusion-driven problem and provided a simple empirical expression for evaporation rate, they also developed a mathematical model to study the internal flow and coupled Marangoni effect in the droplet. Semenov et al. [5] simulated the evaporation process as a quasi-steady state so that the moving boundary problem was converted to a series of fixed boundary problems by calculating the new spherical-cap profile according to the volume reduction. While considering a free-moving surface which is difficult to track in the

\footnotetext{
${ }^{a}$ e-mail: gang.chen@univ-amu.fr
} 
numerical simulation, Yang et al. [8] developed the mathematical model in the frame of Arbitrary Lagrangian-Eulerian (ALE) method. Contrary to the previous works using volume-of-fluid [6] or level-set [7] method, the ALE method allows precisely to track the interfaces of a multi-material system.

The purpose of this work is to investigate a droplet evaporation process on a heated substrate under microgravity conditions. It's a part of numerical studies of the space project EFILE (Space Experiment of Evaporation and Fluid Interfacial Effects). This project is one of the microgravity fluid physics experiments scheduled onboard Chinese scientific satellite SJ-10 [9], aimed to obtain the novel knowledge on the coupling mechanism of evaporation and thermal convection in a system with phase-changed interface in microgravity environment, as well as to better understand the gravity effects on the heat and mass transfers of evaporation.

In the present study, a 2D axisymmetric numerical model for a millimeter size drop that partially wets on a impermeable, heated substrate and evaporates into air is developed. The rest of the paper is organized as follows. The numerical model is described in Section 2. Section 3 presents the numerical procedure used to solve the problem. Then validation of the model is carried out by comparison of the results with those obtained analytically in the literature. Numerical results of evaporation rate, thermal effects of substrate and internal flow field are presented and discussed in Section 4. Finally, conclusions are stated in Section 5.

\section{Numerical model}

We consider a millimeter size droplet evaporating on a heated substrate under the assumption of microgravity conditions. The proposed model consists of three dominant physical phenomena: (1) transport of liquid mass diffusing into the air with a freely moving interface; (2) heat transfer coupled with the non-isothermal phasechanged interface induced by evaporative cooling; and (3) Marangoni flow inside the droplet resulting from surface tension gradient due to temperature variation. A transient (2D) axisymmetric model of sessile liquid droplet is numerically investigated, as shown in Figure 1. We assume that the drop maintains a spherical-cap geometry due to the effect of liquid-gas surface tension $\sigma=\sigma_{0}-\sigma_{T}\left(T-T_{0}\right)$, in which $\sigma_{0}$ and $\sigma_{T}$ are the surface tension at temperature $T_{0}$ and its (negative) gradient with temperature, respectively. Moreover, the buoyancy effect may be neglected in microgravity, i.e. Marangoni effect dominates the whole process. Thermophysical properties of liquids and substrates used in this work are listed in Table 1.

There are two pure modes of evaporation, namely constant-angle and constantradius modes. In the former, the droplet evaporates with receding contact radius $R$ while the contact angle remains fixed at the initial, equilibrium contact angle $\theta=\theta_{0}$. In the latter, the contact angle $\theta$ decreases with time while the base radius remains pinned at $R=R_{0}$. In our ground and space experiments, we used a solid substrate of different radii and injected sufficient liquid volume to make sure that the contact line is well anchored to the substrate with its radius being at the edge of the substrate. Under such conditions, the evaporation process observed follows essentially the constantradius mode during most of the drop's lifetime (90\%). Therefore, it can reasonably be assumed that the contact radius $R$ remains uncharged in our numerical simulations. Nevertheless, to predict the lifetime of droplets evaporating, a more sophisticated numerical mode would be via a combined pinned-receding mode, depending on the wettability of the substrate against the droplet.

The present simulation is a transient simulation, before presenting the problem formulation it is useful to make some comments on the different time scales involved in the present phenomenon. The relevant time scales are the evaporative $\left(m_{0} /|\dot{m}|\right)$, 


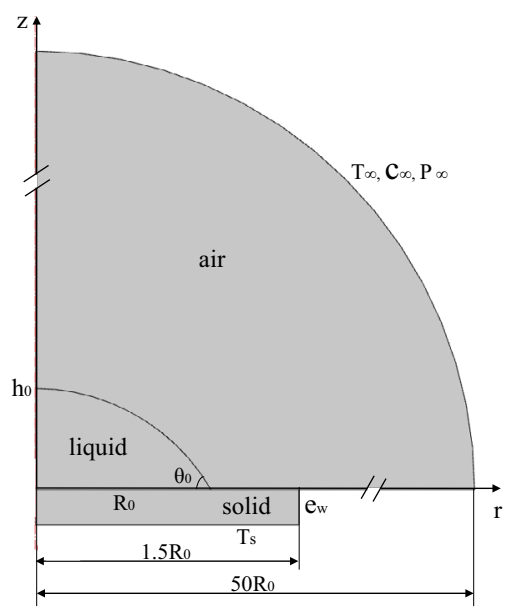

Fig. 1. Computational domain of an axisymmetric sessile droplet on a heated substrate.

Table 1. Thermophysical properties of liquids and substrates used at temperature $T_{\infty}=20^{\circ} \mathrm{C}$ and pressure $P_{\infty}=1 \mathrm{~atm}$.

\begin{tabular}{lccccccc}
\hline Parameter & $\rho\left(\mathrm{kg} / \mathrm{m}^{3}\right)$ & $\mu(\mathrm{Pa} \mathrm{s})$ & $k(\mathrm{~W} / \mathrm{m} \mathrm{K})$ & $c_{p}(\mathrm{~J} / \mathrm{kg} \mathrm{K})$ & $H_{\text {vap }}(\mathrm{kJ} / \mathrm{kg})$ & $\sigma(\mathrm{N} / \mathrm{m})$ & $\sigma_{T}(\mathrm{~N} / \mathrm{m} \mathrm{K})$ \\
\hline Water & 997 & $0.89 \times 10^{-3}$ & 0.6 & 4070 & 2440 & $71.97 \times 10^{-3}$ & $1.657 \times 10^{-4}$ \\
Ethanol & 780 & $1.33 \times 10^{-3}$ & 0.167 & 2444 & 855 & $20.89 \times 10^{-3}$ & $1.2 \times 10^{-4}$ \\
Air & 1.178 & $1.79 \times 10^{-5}$ & 0.025 & 1006 & & & \\
PTFE & 2200 & & 0.25 & 1010 & & & \\
Aluminium & 2700 & & 240 & 900 & & & \\
\hline
\end{tabular}

thermal diffusion $\left(R_{0}^{2} \rho c_{p} / k\right)$, viscous $\left(R_{0}^{2} \rho / \mu\right)$, molecular diffusivity $\left(R_{0}^{2} / D_{0}\right)$ and flow $\left(\mu R / \sigma_{T} \Delta T\right)$ time scales. From our experiments and according to Table 1 , these time scales can be estimated and are on the order of $10^{2}, 10,1,10^{-2}$ and $10^{-3} \mathrm{~s}$, respectively. It's clear that the evaporation time scale is much larger that those of flow and thermal diffusion, we thus can consider the evaporation process at each time as an equilibrium state.

The governing equations for the conservation of mass, momentum and energy are as follows. The diffusion-convection equation for the gas region:

$$
\frac{\partial c}{\partial t}+\mathbf{u} \cdot \nabla c=D_{0} \nabla^{2} c
$$

where $c$ stands for the molar concentration of the liquid vapor, $\mathbf{u}$ is the velocity vector and $D_{0}$ is the diffusion coefficient of vapor in air.

Incompressible Navier-Stokes equations and continuity equation governing the motion in the liquid and air phases are given by

$$
\nabla \cdot \mathbf{u}=0
$$

$$
\rho\left(\frac{\partial \mathbf{u}}{\partial t}+\mathbf{u} \cdot \nabla \mathbf{u}\right)=\nabla \cdot\left[-p \mathbf{I}+\mu\left(\nabla \mathbf{u}+(\nabla \mathbf{u})^{T}\right)\right],
$$

where $\rho, p, \mathbf{I}, \mu$ denote the density, hydrodynamic pressure, identity tensor and dynamical viscosity, respectively.

The energy equation in the whole region (liquid, gas and solid) is given by

$$
\rho c_{p}\left(\frac{\partial T}{\partial t}+\mathbf{u} \cdot \nabla T\right)=k \nabla^{2} T
$$


where $c_{p}$ refers to the specific heat, $T$ is the temperature and $k$ is the thermal conductivity.

These governing equations are subjected to boundary conditions along liquid-gas interface. To begin with, the temperature at the interface is non-uniform based on the latent heat of evaporation, reads

$$
k_{g}(\nabla T)_{g} \cdot \mathbf{n}-k_{l}(\nabla T)_{l} \cdot \mathbf{n}=j_{m} H_{v a p}
$$

where $H_{\text {vap }}$ stands for latent heat of evaporation, $\mathbf{n}$ is the unit normal vector. The subscript $l$ represents the liquid phase, $g$ the air phase, respectively. $j_{m}$ is local evaporation flux, which can be calculated by Fick's law,

$$
j_{m}=-D_{0} \nabla c \cdot \mathbf{n}
$$

For the concentration of vapor $c_{\text {sat }}$ at drop surface, it assumes to be ideal gas and saturated according to the Clausis-Clapeyron equation, defined by

$$
\begin{gathered}
c_{\text {sat }}(T)=\frac{P_{\text {sat }}(T)}{\bar{R} T}, \\
\ln \left(\frac{P_{\text {sat }}}{P_{\text {ref }}}\right)=-\frac{H_{v a p}}{\bar{R}}\left(\frac{1}{T}-\frac{1}{T_{\text {ref }}}\right),
\end{gathered}
$$

where $P_{\text {sat }}$ and $P_{\text {ref }}$ are the pressure of saturated vapor at temperature $T$ and $T_{\text {ref }}$, respectively, $\bar{R}=8.31451 \mathrm{~J} /(\mathrm{mol} \mathrm{K})$ is the universal gas constant.

In particular, the normal and tangential stresses at the liquid-gas boundary should be balanced across and along the interface,

$$
\mathbf{n} \cdot \overline{\mathbf{T}}_{l}-\mathbf{n} \cdot \overline{\mathbf{T}}_{g}=-\sigma(\nabla \cdot \mathbf{n}) \mathbf{n}+\sigma_{T} \nabla_{\Gamma} T
$$

where subscript $\Gamma$ represents the liquid-gas interface, $\overline{\mathbf{T}}$ is the full stress tensor and $\nabla_{\Gamma}$ is the surface gradient operator.

The conservation of mass is as followed so that the velocity for deformed mesh movement can be determined,

$$
\rho_{l}\left(\mathbf{u}_{l} \cdot \mathbf{n}-u_{\Gamma}\right)=j_{m},
$$

where $\mathbf{u}_{l}$ and $u_{\Gamma}$ are liquid and mesh velocity.

The domain size is chosen large enough to minimize boundary effects, typically several ten times larger than the drop. No viscous stress is prescribed at the boundary, which corresponds to an open boundary condition for air flow. One could use a prescribed normal stress condition, e.g. the ambient pressure $p_{\infty}$. Using either of the two boundary conditions finally yields the same evaporation process as long as the size of the surrounding air is chosen large enough.

Since the physical problem we are solving is an unsteady system, initial conditions are also prescribed. At $t=0$, the drop is a spherical cap of height $h_{0}$ and base radius $R_{0}$ (initial contact angle $\theta_{0}$ can be deduced: $\theta_{0}=2 \tan ^{-1}\left(h_{0} / R_{0}\right)$ ); the two-phase flow is at rest $(\mathbf{u}=0)$. The initial pressures in the gas, and within the drop are set to the ambient pressure $\left(p_{\infty}\right)$, and the Laplace pressure $\left(\Delta p=2 \sigma_{0} / R_{s}\right.$ with $R_{s}=$ $\left.\left(h_{0}^{2}+R_{0}^{2}\right) /\left(2 h_{0}\right)\right)$ plus the ambient pressure, respectively. The initial temperature in the whole domain is set to the ambient temperature $T_{\infty}$. The initial concentration of the liquid vapor equals to $c_{\infty}=H c_{s a t}\left(T_{\infty}\right)$, where $H$ is the relative humidity. 


\section{Numerical procedure and validation}

We used a commercial software COMSOL Multiphysics to solve this coupled system of diffusion, fluid flow and heat transfer with moving interface [5]. For a consistent spatial and temporal discretization, the $\mathrm{P} 2+\mathrm{P} 1$ (velocities and pressures are approximated using the second-order and first-order, respectively) and a generalized alpha method are adopted. Furthermore, the ALE method within time-dependent solver allows us to carry out the fully coupled equations on a freely deformed mesh. With the help of an automatic remeshing technique, the distortion of mesh during the unsteady procedure is alleviated. The maximum relative tolerance on the dependent variable $(\mathbf{u}, p, T, c)$ is lower than $0.1 \%$ during the iterative process.

To assess the accuracy of the numerical results, a grid sensitivity analysis has been first undertaken. After several numerical experiments, grids consisting of 18160 elements were selected to control the relative error within $1 \%$. A large size of the surrounding air up to 50 times of droplet radius is used in order to eliminate the boundary effects. The implemented numerical model is validated by comparison of computed results of droplet volume evolution with those obtained by Rowan et al. [10]. As shown in Figure 2a, our numerical results are in very good agreement with their experimental results. Another validation is plotted in Figure 2b, which compares the lifetime of evaporation with the theoretical analysis of Stauber et al. [11]. It is shown that the evolution of evaporation time, $t_{R}$, as a function of the initial contact angle, $\theta_{0}$, is well described by the following integral equation:

$$
t_{R}=\frac{\rho R_{0}^{2}}{D_{0}\left(c_{s a t}-c_{\infty}\right)} \int_{0}^{\theta_{0}} \frac{d \theta}{g(\theta)},
$$

where function $g(\theta)$ is given by

$$
g(\theta)=(1+\cos \theta)^{2}\left\{\tan \frac{\theta}{2}+8 \int_{0}^{\infty} \frac{\cosh ^{2}(\theta \tau)}{\sinh (2 \pi \tau)} \tanh [\tau(\pi-\theta)] d \tau\right\}
$$

\section{Results and discussion}

Numerical simulations are conducted for two different liquid (water \& ethanol) droplets deposited on two different heated substrates (Al \& PTFE). The evaporation occurs in surrounding moist air at relative humidity $(H=50 \%$ for water and $H=0$ for ethanol). Ambient environmental conditions of temperature $T_{\infty}$ equals to $20^{\circ} \mathrm{C}$ and pressure is $1 \mathrm{~atm}$. The bottom surface of substrate is at a prescribed temperature $T_{s}$ varying between $20^{\circ} \mathrm{C}$ and $60^{\circ} \mathrm{C}$.

\subsection{Evaporation rate}

We begin with the evaporation rate. In general, it plays a significant role in heat and mass transfer procedure. The evaporation rate, $\dot{m}$, is defined here as an average total evaporation rate, which is the ratio between the initial volume of the droplet and the lifetime of its evaporation. In other words, it represents a global result and has units of $\mu \mathrm{l} / \mathrm{s}$. In order to gain a deeper insight into the physical phenomenon, various radii of water droplets ranging from 0.6 to $1.4 \mathrm{~mm}$ are considered. The influence of different radii on evaporation rate for a given initial contact angle, $\theta_{0}=50^{\circ}$, is shown in Figure 3. The filled symbols denote the numerical results obtained, while the lines 




(a)



(b)

Fig. 2. Comparison of the numerical results with those reported in the literature: (a) with experimental results by Rowan et al. [10] for water sessile droplet with $\theta_{0}=80^{\circ}$ and different initial radius $R_{0}$, PMMA substrate with $k_{s}=0.18 \mathrm{~W} / \mathrm{m} \mathrm{K}$ and $e_{w}=0.8 \mathrm{~mm}$, ambient environment with $T_{\infty}=21.5^{\circ} \mathrm{C}, H=55 \%$ and $P_{\infty}=1$ atm. The symbols are the literature results and the lines are the present study; (b) with an analysis by Stauber et al. [11] (Eq. (11)). The solid line is for heated Al substrate and the dashed line is for non-heated $\mathrm{Al}$ substrate, the symbols represent our numerical results with water drop at $R_{0}=1 \mathrm{~mm}$, for $T_{\infty}=20^{\circ} \mathrm{C}, H=50 \%$ and $P_{\infty}=1 \mathrm{~atm}$.

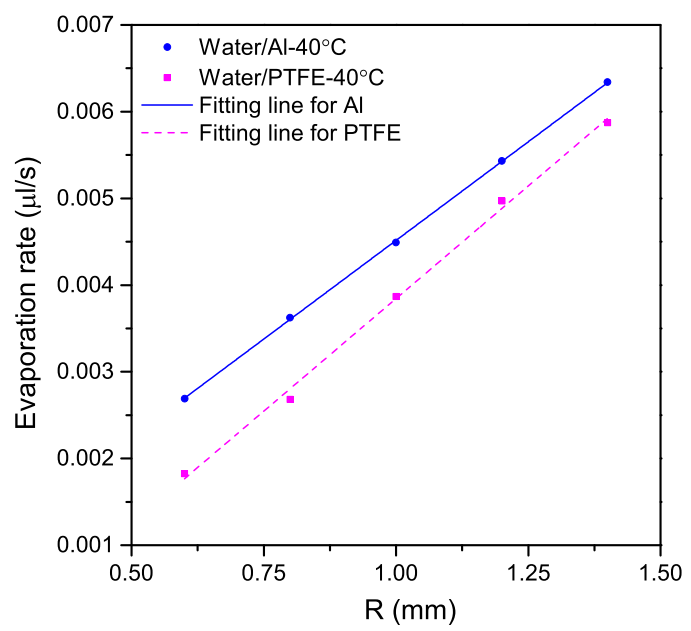

Fig. 3. Evaporation rate as a function of droplet radius for various water droplets evaporated on two substrates (Al and PTFE). The initial value of radius ranges from 0.6 to $1.4 \mathrm{~mm}$ with an initial contact angle $\theta_{0}=50^{\circ}$.

correspond to simple linear regression for each case study. It makes clear a linear scaling of the evaporation rate with the droplet radius, i.e. $|\dot{m}| \sim R$. This finding is consistent with a recent investigation by Carrier et al. [12]. Experimentally, we have also observed such evidence in the system of macroscopic droplets [13]. It is also worth mentioning that the evaporation rate is higher for substrates with better thermal conductor like aluminum (Al). Since Al has higher thermal conductivity than PTFE, 




Fig. 4. Evaporation rate $|\dot{m}|$ of sessile water droplets evaporating on two substrates (Al and PTFE) as a function of the substrate temperature $T_{s} .|\dot{m}|$ follows a power-law scaling with $T_{s}$, i.e. $|\dot{m}|=a T_{s}^{b}$. Al: $a=4.2 \times 10^{-7}, b=2.517$; PTFE: $a=1.1 \times 10^{-6}, b=2.215$.

the evaporation cooling has less effect on $\mathrm{Al}$ substrate. Therefore, the temperature inside the drop is higher and the saturation concentration of vapor at the free surface is closer to the constant value of $c_{s a t}\left(T_{\infty}\right)$, hence the evaporation process taking place faster.

Figure 4 shows the dependence of evaporation rate on the substrate temperature. It is seen that the evaporation rate can be well fitted by a power law with the substrate temperature, i.e. $|\dot{m}| \sim a T_{s}^{b}$. This scaling law has already been reported in several experiments $[14,15]$, but with different power exponent, $b$, for instance, Sobac and Brutin [15] gave a wide range of $b$ (typically in the range between 2.6 and 3) in their experiments, depending upon the thermal conductivity of the substrate being used. In addition, for different droplet sizes, the distinction of evaporation rate is further enhanced with increasing substrate temperature. This may be explained by the enhanced Marangoni convection inside the droplet when the substrate temperature is increased.

\subsection{Thermal effects of substrate}

In what follows, thermal effects of different substrates are discussed by comparison of the temperature profile at the liquid-gas interface. The liquid we use is either water or ethanol, the substrate being either a thermally insulating material (PTFE) or a very good thermal conductor $(\mathrm{Al})$. As shown in Figure 5, at the beginning of the transient period with large contact angle, i.e. $\theta_{0}=35^{\circ}$, the temperature distribution along the interface is dominated by the evaporation cooling effect for all the cases, representing the coldest region appears at the drop apex. During evaporation, different patterns of the surface temperature distribution along the droplet surface, depending on the substrate. For a substrate with higher thermal conductivity like Al, as indicated in Figure 5b and Figure 5d, the surface temperature always increases from the center to the edge of the drop, Marangoni effect brings the hot liquid to the cold area reducing the temperature difference, while for an insulating substrate PTFE, the temperature exhibits a non-monotonic spatial distribution along the droplet surface. Thermal resistance is so small that the surface temperature is more likely dominated 


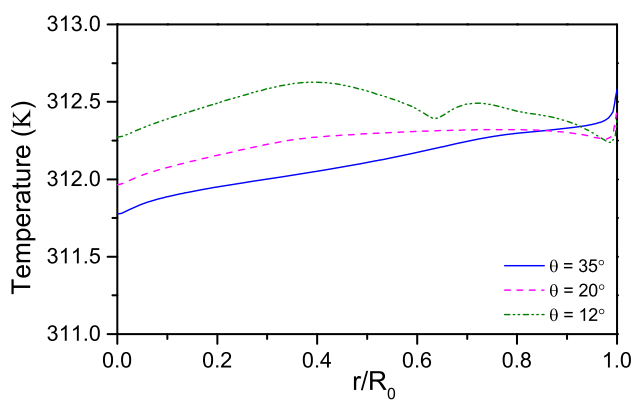

(a) water-PTFE

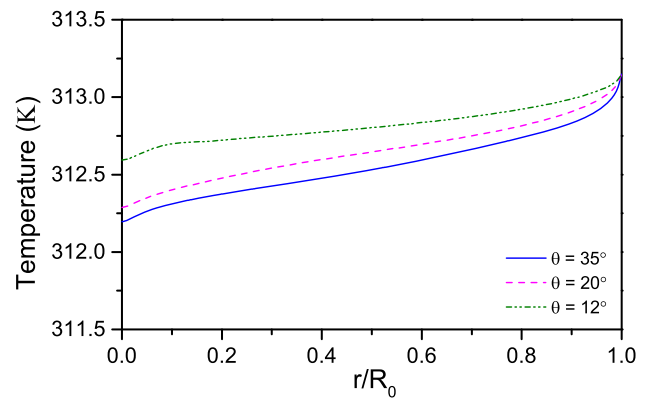

(b) water-Al

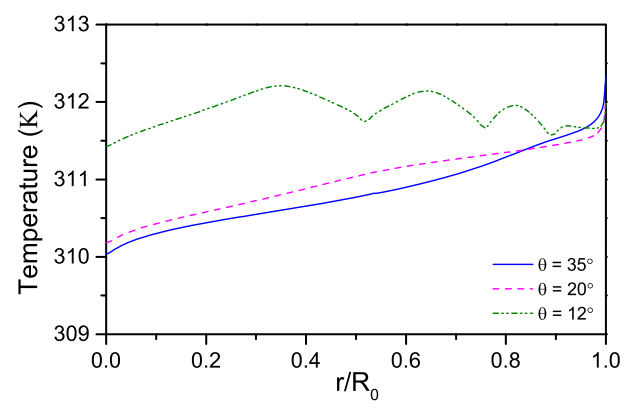

(c) ethanol-PTFE

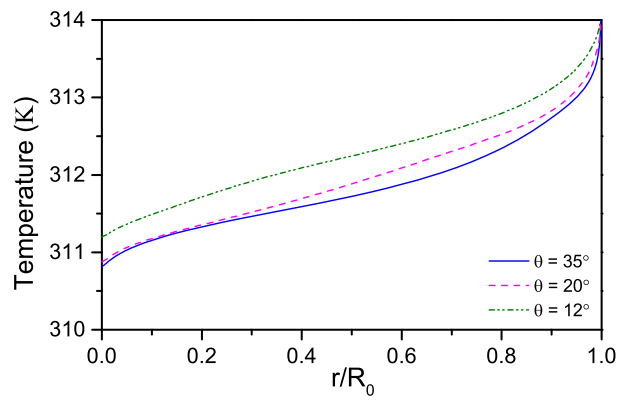

(d) ethanol-Al

Fig. 5. Temperature profile at the liquid-gas interface at different stages of evaporation (denoted by instantaneous contact angle, $\theta$ ) for different liquid and different substrates, for an initial contact angle $\theta_{0}=41^{\circ}$ and the substrate temperature $T_{s}=40^{\circ} \mathrm{C}$.

by the conduction in the liquid phase. It is interesting to mention that in this case the temperature gradient near the triple line is inverted when the contact angle becomes very small, because most of the evaporation occurs at triple line, as a result, the temperature distribution is lower near the triple line. On the other hand, a comparison of the different type of liquids is required to understand the flow pattern induced by Marangoni effect. From Figure $5 \mathrm{a}$ and Figure 5c, we can conclude that for a more volatile liquid like ethanol, the non-monotonic temperature distribution is more unstable, indicating that the liquid motion behavior inside the droplet becomes more active and complex, as also evidenced in the following subsection.

\subsection{Internal flow field}

Figure 6 illustrates the flow structure inside an ethanol droplet at the onset of multicellular due to Marangoni effect. It is found that when the contact angle decreases below a certain value about $13^{\circ}$, the non-monotonic distribution of the temperature at free surface generates multiple clockwise and counterclockwise convective cells. This can be explained by the Marangoni stress along the droplet surface generated by this non-monotonic surface temperature profile. At initial stage of evaporation, the surface temperature decreases monotonically from the center to the edge of the droplet, an inward stress generates a counterclockwise circulation inside the droplet. If the distribution of the surface temperature is non-monotonic, as plotted in Figure $5 \mathrm{c}$, each positive (negative) temperature gradient along the droplet surface gives rise to 


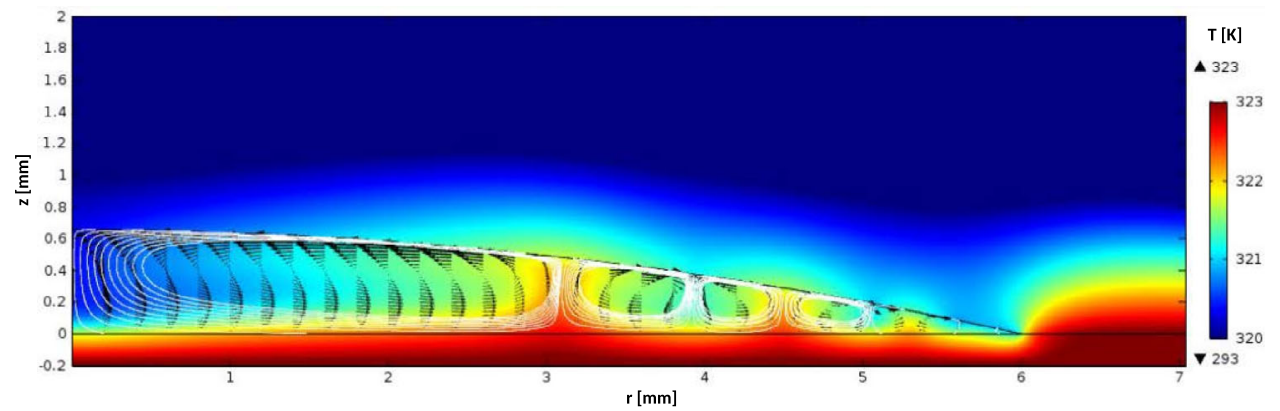

Fig. 6. Multicellular thermocapillary flow pattern at $\theta=13^{\circ}$ within an ethanol droplet evaporating on a PTFE substrate. Initial conditions: $\theta_{0}=41^{\circ}, R_{0}=6 \mathrm{~mm}, T_{\infty}=20^{\circ} \mathrm{C}$, $T_{s}=50^{\circ} \mathrm{C}, H=0$, and $P_{\infty}=1 \mathrm{~atm}$. Velocity field in the droplet is represented by streamlines and arrows, temperature field by colour plot.

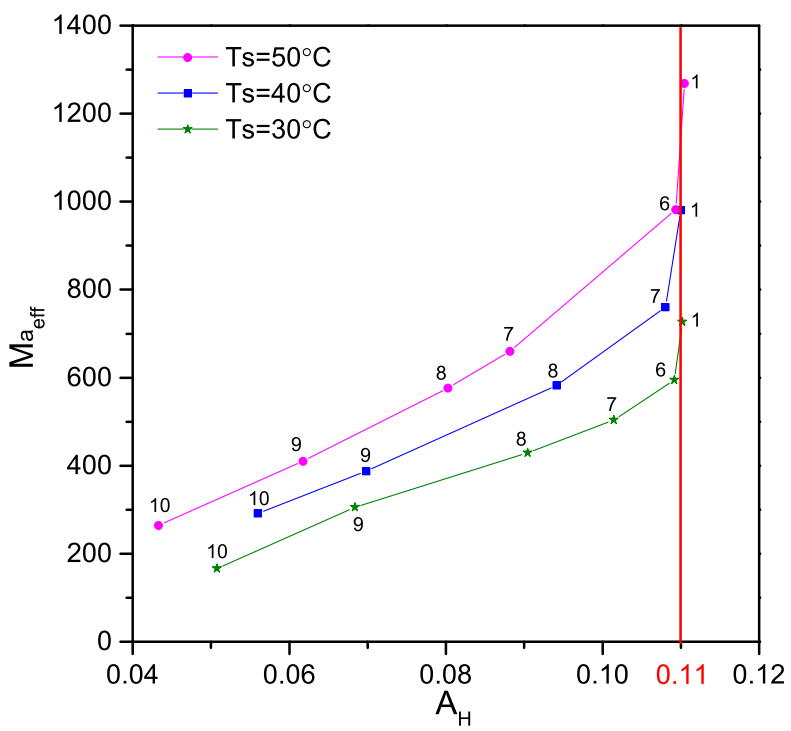

Fig. 7. Variation of the effective Marangoni number of an ethanol droplet as a function of the geometric aspect ratio $A_{H}=h / R$. The indicated number is the number of convective cells presented within the droplet when $A_{H}$ is decreased below a threshold, i.e. 0.11.

an inward (outward) Marangoni stress, thereby leading to a multicellular thermocapillary flow pattern $[16,17]$.

In order to get more insight into the thermocapillary convection within the evaporating drop, we define an effective Marangoni number, denoted by $M a_{e f f}=\frac{\sigma_{T} \Delta T h}{\mu \alpha}$, where $h$ is the (time-dependent) height of drop apex, $\triangle T$ refers to the temperature difference between the drop apex and the base of the droplet and $\alpha$ is thermal diffusivity, respectively. Figure 7 summarizes the variation of this effective Marangoni number as a function of the geometric aspect ratio $A_{H}=h / R$. The multicellular thermocapillary flow pattern occurs when the geometric aspect ratio is decreased below a threshold $A_{H} \approx 0.11$. Therefore, this geometric parameter controls essentially the multicellular flow behavior. Furthermore, the number of thermal cells $m$ is closely connected with $A_{H}$ as found in different configurations [18]. It is found 
in our simulations that a relationship holds between the number of thermal cells $m$ and the geometric aspect ration $A_{H}: m A_{H} \approx 0.5-0.7$ for an ethanol droplet. However, we must stress that this relationship is obtained for an axisymmetric pinned droplet.

\section{Conclusions}

The evaporation of a millimeter size droplet that partially wets on heated substrate with pinned contact line has been numerically investigated. Numerical simulations were based on a transient 2D axisymmetric model, taking into account the mass transport, convective flow and heat conduction as well as moving interface. Numerical results revealed that the evaporation rate scales linearly with droplet radius and follows a power-law scaling with substrate temperature. It is highlighted that thermal effect of the substrate has a great impact on the temperature profile at the drop surface, which leads to a type of multicellular thermocapillary flow pattern, especially at the end of evaporation. In particular, the structure of the multicellular flow pattern induced within a heated drop is mainly depending on a geometric aspect ratio. A relationship between the number of convective cells and the aspect ratio was found. Further development of extension 2D axisymmetric model into a 3D model is needed for a better understanding of the instability problem of droplet evaporation and the work is in progress.

We would like to thank S. Semenov for helpful discussions. This work was financially supported by the National Natural Science Foundation of China (Grants No. 11532015, No. 11302236), by the Strategic Priority Research Program on Space Science, Chinese Academy of Sciences (Grants No. XDA04073000, XDA04020202-02), and by the CNES (Centre National d'Études Spatiales). The work was conducted within the framework of a joint Doctoral Training Program (DPP) between Chinese Academy of Sciences (CAS) and Centre National de la Recherche Scientifique (CNRS). Funding from the CAS and support from the CNRS/CNES are gratefully acknowledged.

\section{References}

1. H.Y. Erbil, Adv. Colloid Interface Sci. 170, 67 (2012)

2. Z.Q. Zhu, D. Brutin, Q.S. Liu, Y. Wang, A. Mourembles, J.C. Xie, L. Tadrist, Microgravity Sci. Technol. 22, 339 (2010)

3. R. Liu, O.A. Kabov, Phys. Rev. E 85, 066305 (2012)

4. H. Hu, R.G. Larson, J. Phys. Chem. B 106, 1334 (2002)

5. S. Semenov, V.M. Starov, R.G. Rubio, Eur. Phys. J. Special Topics 219, 143 (2013)

6. L. Adil, J. Ouazzani, M. Antoni, Colloids Surfaces A Physicochem. Eng. Aspects 460, $342(2014)$

7. J. Chen, Z.H. Wang, C. Yang, Z.S. Mao, Chem. Eng. Technol. 38, 155 (2015)

8. K. Yang, F.J. Hong, P. Chen, Int. J. Heat Mass Transfer 70, 409 (2014)

9. W.R. Hu, J.F. Zhao, M. Long, X.W. Zhang, Q.S. Liu et al., Microgravity Sci. Technol. 26, 159 (2014)

10. S.M. Rowan, G. McHale, M.I. Newton, J. Phys. Chem 99, 13268 (1995)

11. J.M. Stauber, S.K. Wilson, B.R. Duffy, K. Sefiane, J. Fluid. Mech 744, R2 (2014)

12. O. Carrier, N.S. Bonn, R. Zargar, M. Aytouna, M. Habibi, J. Eggers, D. Bonn, J. Fluid. Mech. 798, 774 (2016)

13. X. Chen, Z.Q. Zhu, Q.S. Liu, X.W. Wang, Microgravity Sci. Technol. 26, 159 (2016)

14. S.A. Putnam, A.M. Briones, L.W. Byrd, J.S. Ervin, M.S. Hanchak, A. White, J.G. Jones, Int. J. Heat Mass Transfer 55, 5793 (2012) 
15. B. Sobac, D. Brutin, Phys. Rev. E 86, 021602 (2012)

16. G. Lu, Y.Y. Duan, X.D. Wang, D.J. Lee, Int. J. Heat Mass Transfer 54, 4437 (2011)

17. C. Bouchenna, M.A. Saada, S. Chikh, L. Tadrist, Interfacial Phenomena and Heat Transfer 3, 185 (2015)

18. G. Chen, A. Lizée, B. Roux, J. Cryst. Growth 180, 238 (1997) 